\title{
Assessment of left ventricular mechanical dyssynchrony in coronary artery disease
}

\author{
Chetan D. Patel, $M D{ }^{a}$ and Anirban Mukherjee, $M D^{a}$ \\ a Department of Nuclear Medicine, All India Institute of Medical Sciences, New Delhi, India
}

Received Aug 21, 2015; accepted Aug 21, 2015

doi: $10.1007 / \mathrm{s} 12350-015-0276-3$

\section{See related article, pp. 728-736}

The assessment of left ventricular mechanical dyssynchrony (LVD) using phase analysis of gated myocardial perfusion SPECT (MPS) was introduced in 2005, allowing for the simultaneous assessment of left ventricular $(\mathrm{LV})$ perfusion, function, and mechanical dyssynchrony. ${ }^{1}$ Phase analysis has shown excellent reproducibility and repeatability for assessing LVD. ${ }^{2}$ Also, compared to other imaging modalities, such as echocardiography, magnetic resonance imaging, and equilibrium radionuclide angiography, phase analysis of MPS has shown several advantages, such as simplicity, widespread availability, superior reproducibility, applicability to retrospective data, and ability to simultaneously assess myocardial scar location and severity for optimizing cardiac resynchronization therapy (CRT) in heart failure patients. ${ }^{3,4}$ Aljaroudi $^{5}$ outlined other potential clinical applications of LVD including prognostication and risk stratification of patients with ischemic, ${ }^{6}$ non-ischemic cardiomyopathy, ${ }^{7}$ implantable defibrillators ${ }^{8}$, and end-stage renal disease. $^{9,10}$

Several studies have demonstrated the relation of LVD with myocardial ischemic and/or scar burden, ${ }^{11-13}$ coronary artery disease (CAD) extent ${ }^{14}$, and LV contractile function. ${ }^{15}$ However, there are conflicting data in the literature about the effect of different patterns of perfusion abnormality on LVD in CAD patients. Aljaroudi et $\mathrm{al}^{12}$ in their study on CAD patients who

Reprint requests: Chetan D. Patel, MD, Department of Nuclear Medicine, All India Institute of Medical Sciences, B-54, South Extension Part-1, New Delhi, 110049, India; chetantruptipatel@hotmail.com J Nucl Cardiol 2016;23:737-40.

$1071-3581 / \$ 34.00$

Copyright (c) 2015 American Society of Nuclear Cardiology. underwent stress-rest Tc99m-sestamibi, reported that the rest and stress phase-derived standard deviation (PSD) and the rest and stress bandwidth (PHB) were similar in patients with reversible perfusion defect compared to patients with normal perfusion and that presence of even large reversible perfusion defects did not alter the indices of mechanical dyssynchrony on MPS. This was attributed to delayed image acquisition after stress in Tc99m-sestamibi study. So it was proposed, that stressinduced changes in LVD may be better evaluated using thallium-201 (Tl-201) MPS, as the post-stress images are acquired within 10 minutes of stress in Tl-201 study. Chen et $\mathrm{al}^{13}$ evaluated this aspect in their study on 75 patients who underwent stress-rest Tl-201 MPS. The authors suggested that stress-induced ischemia causes dyssynchronous contraction in the ischemic region, leading to worsening of LVD whereas normal myocardium showed more synchronous contraction during stress. However, in a similar study, Singh et $\mathrm{al}^{11}$ found that LVD parameters were smaller on post-stress as compared to rest study on Tl-201 MPS, regardless of perfusion pattern. Stress-induced worsening of LVD was observed only in patients with perfusion abnormalities, but this is not related to the type of perfusion abnormality. Therefore, there is still a lack of clarity on the exact relationship of the LVD with the type of perfusion abnormalities on MPS performed with different radiotracers and study protocol. There are limited publications on prognostic value of LVD in patients with CAD. ${ }^{15,16}$ Zafrir et al ${ }^{15}$ in a study group of 787 patients followed for mean duration of $18.3 \pm 6.2$ months observed that LVD can successfully predict cardiac death together with NYHA class, in patients with LV dysfunction.

In this issue of the Journal of Nuclear Cardiology, Gimelli et $\mathrm{al}^{17}$ have evaluated the relative interactions between coronary anatomy, myocardial perfusion, and LV function with LVD in patients with known or suspected CAD. Although LVD can be an important parameter in predicting adverse prognosis in patients 
with $\mathrm{CAD}$, this article has not evaluated the prognostic implication but merely observed the prevalence of LVD and evaluated its determinants in these patients. The authors studied 657 consecutive patients with angina pain and known or suspected CAD who underwent Tc99m-Tetrofosmin MPS on a dedicated cardiac camera equipped with cadmium-zinc-telluride (CZT) detectors. Further, the coronary anatomy was assessed by either CT or invasive coronary angiography. A coronary stenosis $\geq 70 \%$ was considered significant. Summed stress score (SSS), sum rest score (SRS), and summed difference score (SDS) were calculated. The authors also quantified end diastolic volume (EDV), end systolic volume (ESV), ejection fraction (EF), and peak filling rate (PFR) both for the rest and the stress studies. However, only resting LV dyssynchrony was determined in this study.

Cardiac cameras equipped with CZT detectors allow myocardial perfusion imaging at a reduced radiation burden and acquisition time with a significantly improved spatial resolution. ${ }^{18,19}$ However, the values of PSD and PHB as a marker of dyssynchrony indices may change with conventional vs. CZT cameras, different radiotracers, study protocol, and software used for dyssynchrony assessment and in different population. Appropriately, the authors in this study, defined the normal cut-off values of PSD and PHB from 'normal' sub-group comprising patients with normal LVEF $(>55 \%)$, normal coronary anatomy, and normal MPS. Thus, defining the cut-offs values which are imaging system, quantitative software (Corridor4DM), and population specific. The normal cut-off values for PSD and PHB (at rest) in this study are \{men (bandwidth $89^{\circ} \pm 38^{\circ} ; \mathrm{SD} 20^{\circ} \pm 10^{\circ}$ ) and women (bandwidth $88^{\circ} \pm 31^{\circ} ;$ SD $21^{\circ} \pm 11^{\circ}$ ) .

In our experience, the normal cut-off values of PSD and PHB on Tc99 m-sestamibi derived on our own population \{men (bandwidth $30.6 \pm 7.6$; SD $8.9 \pm 2.9$ ) and women (bandwidth $25.3 \pm 8.6$; SD $7.7 \pm 2.7$ ) (unpublished data) were different from the LVD parameters reported by Chen et $\mathrm{al}^{1}$ \{men (bandwidth $38.7 \pm 11.8 ;$ SD $14.2 \pm 5.1$ ) and women(bandwidth $30.6 \pm 9.6$; SD $11.8 \pm 5.2$ ) $\}$ using Tl-201/Tc-99m sestamibi rest/exercise study. Both studies were performed on conventional cameras using same quantitative software ('SyncToolTM' Emory Cardiac Toolbox). But after taking into consideration other factors like dose of radiotracer and study protocol, the study population was found to be themost important reason for the difference in normal cut-off values of PSD and PHB. One possible explanation is that Asian population usually have a lower BMI as compared to people in the western population. ${ }^{20}$ The effect of BMI is of particular importance since people with larger BMI will have more attenuation and less counts. Lesser the counts per pixel, the higher is the noise and potential measurement errors leading to higher PSD and PHB indices. ${ }^{21}$ Interestingly, the normal cut-off values derived by Chen et $\mathrm{al}^{1}$ and our values are markedly different and lower than the values obtained by Gimelli et $\mathrm{al}^{17}$ in this study which was performed on CZT system using different study protocols and quantitative software. Given these variables, it is more ideal to establish the normal cut-off values of synchrony based on specific radiotracer, dose of radiotracer, study protocol, quantitative software, and preferably specific population. The normal cut-off values of synchrony are also dependent on selection criteria of 'normal group' from which these values are derived. ${ }^{22}$

In the present study by Gimelli et al, ${ }^{17}$ significant CAD ( $\geq 70 \%$ on CT/invasive angiography) was present in 415/657 (63\%) patients of which 187 (45\%) showed LVD. In remaining $242(37 \%)$ patients with non-obstructive CAD, LVD was present in $60(25 \%)$ patients. The prevalence of LVD in patients with non-obstructive CAD on angiography is high in this study population. Although, 415 (63\%) patients had significant obstructive CAD on angiography, $567(86 \%)$ patients had abnormal MPS (scar/ischemia/scar \& ischemia). By convention these positive MPS results in 152 patients would be considered false positive. While LVD was present in 10/ 90 patients (11\%) with normal MPS, it was noted in 237/ $567(42 \%)$ patients with abnormal MPS. So overall, out of 247 patients with LVD, 50 patients with LVD had abnormal MPS but non-significant CAD on angiography. The authors have, therefore, observed a stronger association between the presence of LVD and perfusion abnormalities on MPS, even in the absence of significant $\mathrm{CAD}$ on $\mathrm{CT}$ or conventional angiography. But, can the presence of dyssynchrony in this subset of patient be considered as a marker of early ischemia is uncertain. A large prospective trial with long-term follow-up for cardiac events could resolve this issue and provide guidelines for management.

Stress-induced worsening of LVD is a reflection of myocardial stunning and, therefore, a marker for multivessel disease (MVD). Detection of stress-induced worsening of LVD is dependent on the time interval between peak stress and imaging. ${ }^{5}$ Post-stress images in Tl-201 study are acquired within 10 minutes of peak stress and, therefore, it is possible to detect post-stress LVD. Huang et $\mathrm{al}^{14}$ observed that patients with MVD had significantly more global and territorial dyssynchrony at early post-stress than at rest on Tl-201 study. On the other hand, in Tc99m-labeled MPS post-stress imaging usually begins 30-60 minutes after the peak stress, often resulting in the recovery of stress-induced worsening of LVD. So post-stress worsening of LVD, may often be missed during Tc99m-labeled MPS. 
Interestingly, Gimelli et $\mathrm{al}^{17}$ on Tc99m-tetrofosmin study, observed higher prevalence of MVD in patients with LVD at rest than those without dyssynchrony. Since post-stress LVD was not determined in this study, it is difficult to assess the relation of MVD with poststress or change in the post-stress and rest LVD parameters. Relation between dyssynchrony at rest on Tc99m-labeled MPS and MVD needs further validation and if established can serve as a marker for MVD.

In addition, the post-stress LVD could have provided more information on any post-stress alteration of dyssynchrony and its relation with the perfusion abnormality and other functional parameters, especially since this study was performed on CZT camera which has better count statistics (for relatively low-dose stress study) and as per protocol followed in this study, the stress images were acquired within 10-15 minutes of stress injection.

The study further investigated the interactions among myocardial functional parameters at rest (EDV \& ESV index, PFR, EF), perfusion parameters (SRS, SDS) and significant CAD ( $\geq 70 \%)$ and LVD derived on rest study. All these parameters showed significant association on univariate analysis. On multivariate analysis, the resting ESV index and SDS remained only independent predictors of LVD, overwhelming the effects of CAD extent and of LV systolic dysfunction. The authors have suggested that association of these parameters with LVD at rest might allow a better risk stratification of anginal patients, possibly helping clinical decision making.

The retrospective study by Gimelli et al has demonstrated the prevalence of LVD and its determinants in patients with known or suspected CAD. Its importance can be known only, after its prognostic implication and, therefore, its value in the management of the CAD patients is proved. A large prospective trial revealing its prognostic utility can translate LV dyssynchrony into clinically important parameter and add a new dimension in gated MPS study.

\section{Acknowledgment} study.

No funding was received from any organization for the

\section{Disclosure}

The authors declare no conflict of interest.

\section{References}

1. Chen J, Garcia EV, Folks RD, Cooke CD, Faber TL, Tauxe EL, et al. Onset of left ventricular mechanical contraction as determined by phase analysis of ECG gated myocardial perfusion SPECT imaging: Development of a SPECT myocardial perfusion imaging diagnostic tool for assessment of cardiac mechanical dyssynchrony. J Nucl Cardiol 2005;12:687-95.

2. Trimble MA, Velazquez EJ, Adams GL, Honeycutt EF, Pagnanelli RA, Barnhart HX, et al. Repeatability and reproducibility of phase analysis of gated SPECT myocardial perfusion imaging used to quantify cardiac dyssynchrony. Nucl Med Commun 2008;29:37481.

3. Boogers MJ, Chen J, van Bommel RJ, Borleffs CJ, Dibbets-Schneider P, van der Hiel B, et al. Optimal leftventricular leads position assessed with phase analysis on gated myocardial perfusion SPECT. Eur J Nucl Med Mol Imaging 2011;38:230-8.

4. Chen J, Garcia EV, Bax JJ, Iskandrian AE, Borges-Neto S, Soman P. SPECT myocardial perfusion imaging for the assessment of left ventricular mechanical dyssynchrony. J Nucl Cardiol 2011;18:685-94.

5. Aljaroudi W. Early post-stress LV dyssynchrony: A new marker for significant CAD. J Nucl Cardiol 2014;21:1057-61.

6. Aljaroudi W, Alraies MC, Hachamovitch R, Jaber WA, Brunken $\mathrm{R}$, Cerqueira MD, et al. Association of left ventricular mechanical dyssynchrony with survival benefit from revascularization: A study of gated positron emission tomography in patients with ischemic LV dysfunction and narrow QRS. Eur J Nucl Med Mol Imaging 2012;39:1581-91.

7. Goldberg AS, Alraies MC, Cerqueira MD, Jaber WA, Aljaroudi WA. Prognostic value of left ventricular mechanical dyssynchrony by phase analysis in patients with non-ischemic cardiomyopathy with ejection fraction $35-50 \%$ and QRS $<150 \mathrm{~ms}$. J Nucl Cardiol 2014;21:57-66.

8. Aljaroudi WA, Hage FG, Hermann D, Doppalapudi H, Venkataraman R, Heo J, et al. Relation of left-ventricular dyssynchrony by phase analysis of gated SPECT images and cardiovascular events in patients with implantable cardiac defibrillators. J Nucl Cardiol 2010;17:398-404.

9. Aggarwal H, Aljaroudi WA, Mehta S, Mannon R, Heo J, Iskandrian AE, et al. The prognostic value of left ventricular mechanical dyssynchrony using gated myocardial perfusion imaging in patients with end-stage renal disease. J Nucl Cardiol 2014;21:73946.

10. Aljaroudi W, Aggarwal H, Venkataraman R, Heo J, Iskandrian $\mathrm{AE}$, Hage FG. Impact of left ventricular dyssynchrony by phase analysis on cardiovascular outcomes in patients with end-stage renal disease. J Nucl Cardiol 2010;17:1058-64.

11. Singh H, Patel CD, Sharma P, Naik N, Singh S, Narang R. Does perfusion pattern influence stress-induced changes in left ventricular mechanical dyssynchrony on thallium-201-gated SPECT myocardial perfusion imaging? J Nucl Cardiol 2015;22:36-43.

12. Aljaroudi WA, Koneru J, Heo J, Iskandrian AE. Impact of ischemia on left ventricular dyssynchrony by phase analysis of gated single photon emission computed tomography myocardial perfusion imaging. J Nucl Cardiol 2011;18:36-42.

13. Chen CC, She TY, Chang MC, Hung GU, Chen WC, Kao CH, et al. Stress-induced myocardial ischemia is associated with early post-stress left ventricular mechanical dyssynchrony as assessed by phase analysis of 201Tl gated SPECT myocardial perfusion imaging. Eur J Nucl Med Mol Imaging 2012;39:1904-9.

14. Huang W, Huang C, Lee C, Chem C, Chuang-Uei H, Chen J. Relation of early post-stress left ventricular dyssynchrony and the extent of angiographic coronary artery disease. J Nucl Cardiol 2014;21:1048-56

15. Zafrir N, Nevzorov R, Bental T, Strasberg B, Gutstein A, Mats I, et al. Prognostic value of left ventricular dyssynchrony by myocardial perfusion-gated SPECT in patients with normal and abnormal left ventricular functions. J Nucl Cardiol 2014;21:532-40. 
16. Uebleis C, Hellweger S, Laubender RP, Becker A, Sohn HY, Lehner S, et al. Left ventricular dyssynchrony assessed by gated SPECT phase analysis is an independent predictor of death in patients with advanced coronary artery disease and reduced left ventricular function not undergoing cardiac resynchronization therapy. Eur J Nucl Med Mol Imaging 2012;39:1561-9.

17. Gimelli A, Liga R, Giorgetti A, Favilli B, Pasanisi EM, Marzullo P. Determinants of left ventricular mechanical dyssynchrony in patients submitted to myocardial perfusion imaging: a cardiac CZT study. J Nucl Cardiol 2015. doi:10.1007/s12350-015-0247-8.

18. Gimelli A, Bottai M, Giorgetti A, Genovesi D, Kusch A, Ripoli A, et al. Comparison between ultrafast and standard single-photon emission CT in patients with coronary artery disease: a pilot study. Circ Cardiovasc Imaging 2011;4:51-8.
19. Giorgetti A, Masci PG, Marras G, Rustamova YK, Gimelli A, Genovesi D, et al. Gated SPECT evaluation of left ventricular function using a CZT camera and a fast low-dose clinical protocol: comparison to cardiac magnetic resonance imaging. Eur J Nucl Med Mol Imaging 2013;40:1869-75.

20. WHO Expert Consultation. Appropriate body-mass index for Asian populations and its implications for policy and intervention strategies. Lancet 2004;363:157-63.

21. Saha G. Statistics of radiation counting. In: Saha G, editor. Physics and radiobiology of nuclear medicine. 3rd ed. New York: Springer; 2006. p. 34-43.

22. Hage FG, Garcia EV. The independent prognostic value of left ventricular dyssynchrony. J Nucl Cardiol 2014;21:541-3. 